\title{
Some Considerations on Diagnosis in Leprosy and on the Treatment of Lepers.
}

\author{
T. B. WeLCH.
}

(Reprinted, by kind permission of the editor, from " East African Medical Journal," Vol. 2, No. 3, June, 1934).

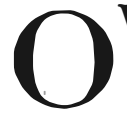

VER-OPTIMISTIC reports circulated from time to time, subsequently exaggerated, have done a great deal to injure the prestige of modern methods in the treatment of lepers. Further damage has been done by the application of these methods to patients hopelessly involved. Valuable though these methods are, their indiscriminate application is to be deprecated, and it behoves those undertaking such treatment to exercise a judicious discrimination in their selection of patients.

I propose to-day to stress the importance of adequate diagnosis and treatment, and particularly to emphasise the fact that the leper is rarely only a leper-a fact unfortunately often overlooked, and so such prospects of success as may be present are rendered remote.

The leper whose disease is clinically only recognisable is in great danger, however slight its manifestations ; in much greater peril is he in whose tissues $M$. leprae are found, while the more readily they are demonstrated the more precarious is his condition. Nevertheless, in a very considerable number of early cases and in a fair number of those that are only moderately advanced, arrest of leprosy without residual deformity follows careful attention to the making of a full diagnosis and to the provision of adequate treatment. In still more advanced cases arrest may similarly be obtained but with the persistence of more or less residual deformity. Little, however, is to be expected of more particularly anti-leprotic measures unless the patient is in fairly good general condition before bringing them into use ; if such condition cannot be attained, these measures, helpful as they are within their limited field, should be withheld, since their use, inevitably followed by failure, tends to bring them into unmerited disrepute because their limitations have not been duly appreciated.

$M$. leprae establishes itself readily in children; less readily in adolescents, and with difficulty in otherwise healthy adults, the difficulty increasing with advancing years. Successful invasion may, however, take place in adults whose resistance is lowered by one or more predisposing causes, though usually only after prolonged and 
close association with another leper. Such causes may be transient, such as acute illness; or they may be of longstanding, such as chronic disease, habitual under-feeding, an ill-balanced diet, and so on.

Not only does $M$. leprae become established with difficulty, but also it shows a definite tendency to die out. Abortive cases, in which leprosy is manifested by one or two macules only, that have remained unchanged for years, are not rare ; cases in which progress has been exceedingly slow and in which elimination has ultimately taken place, leaving the patient comparatively undamaged are more common; while even more frequent are cases that have passed on to the non-infective stage of late neural leprosy with residual mutilation after a prolonged course. In all these cases, successful invasion has been followed sooner or later by the dying off of the invaders.

Since normally healthy persons living under good conditions, rarely, if ever, acquire leprosy, and since some ascertainable predisposing cause usually, and perhaps always, precedes successful invasion by $M$. leprae, it seems possible that the removal of such causes may contribute to the arrest of leprosy, and this is found to be so in practice. In some cases of early leprosy such removal may be all that is required, while in more advanced cases a good deal may be expected of more particularly anti-leprotic measures after any other unfavourable conditions present have been set right.

It is important to remember that often there is more than one predisposing cause present. Thus ankylostomiasis and some dietetic deficiency such as hypovitaminosis frequently occur in the same patient.

The importance of dietetic deficiency in relation to leprosy is gaining increasing recognition. Muir (1932) states that in twenty-nine early cases he has brought about arrest of leprosy by putting the patients on a properly balanced diet. Dietetic deficiency is, however, not uncommon in institutions, while it is common in lepers at large, and it is apt to be overlooked. The importance of some factor is easily exaggerated at the expense of others of no lesser significance; but it is probable that syphilis or ankylostomiasis often in the presence of some dietetic deficiency are by far the most usual causes predisposing the leprosy.

That diet might be a factor in determining the incidence of leprosy was suggested long before these days of progress in rational dietetics. Gilbert White (1778) in a letter later included in The Natural History of Selborne (1789) described 

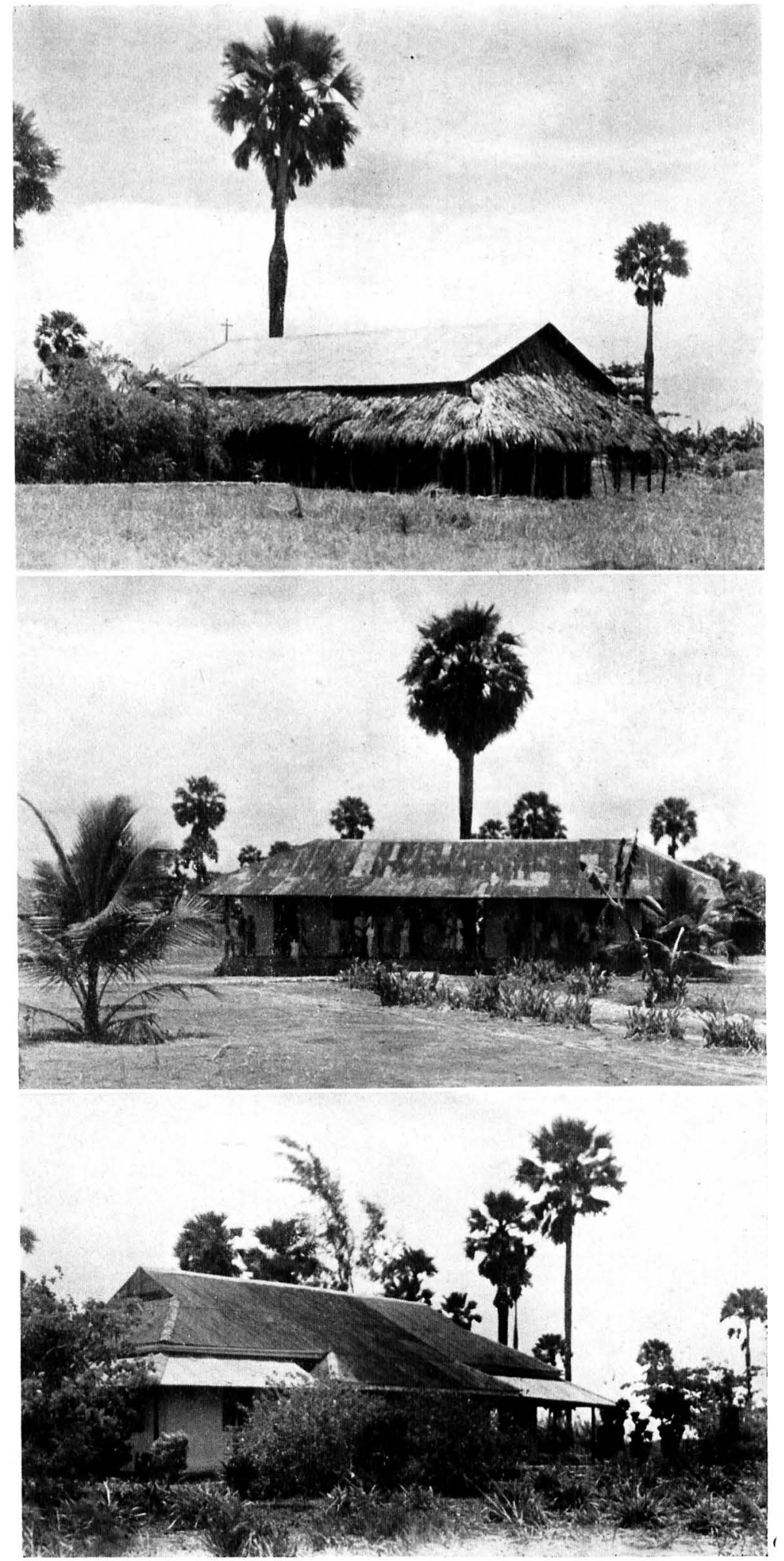

FUNZI LIPROSY SETTILMIENT, ZANZIBAR.

1 - 
No. 5

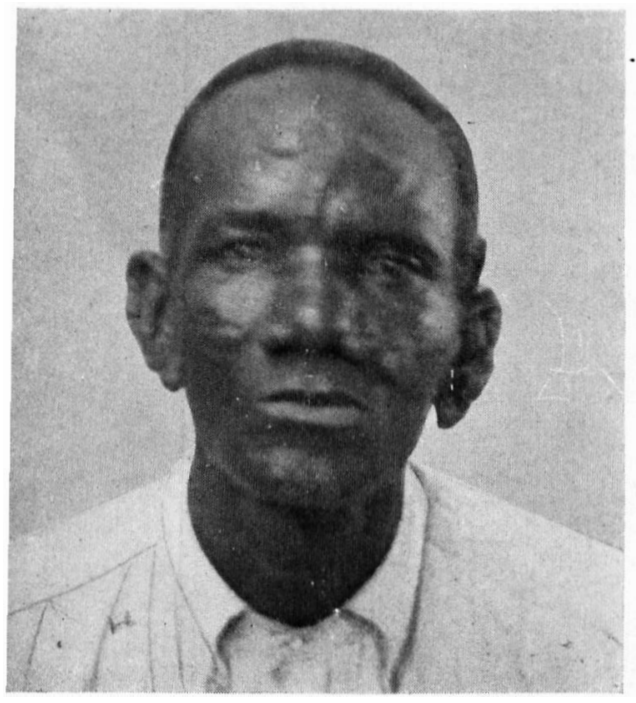

C.3 Case. On Admission
No. 5

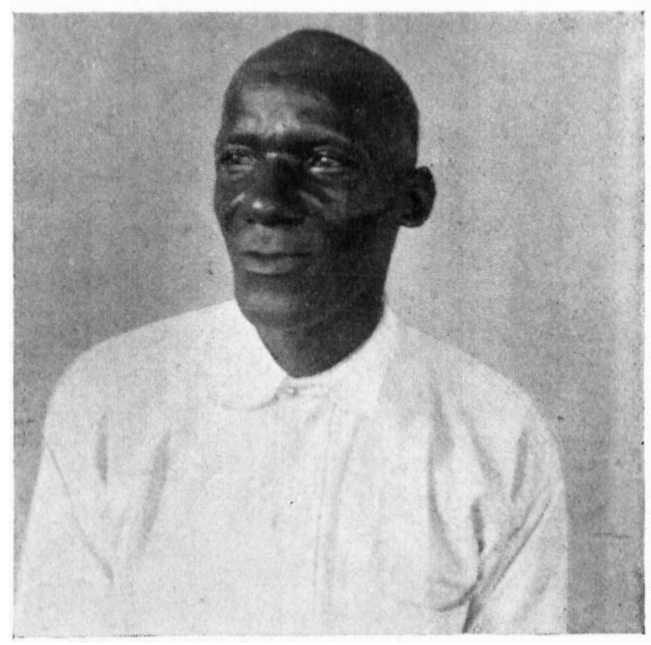

After 3 lears

THE CURABILITY OF LEPROSY

io. 6

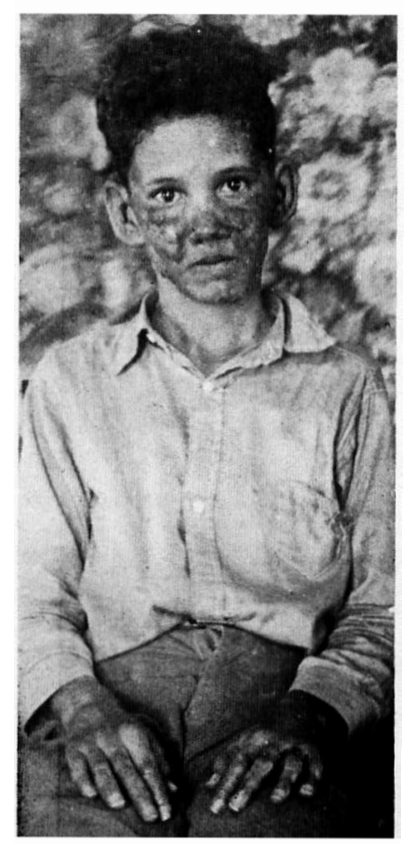

$\mathrm{C}_{3} \mathrm{Nr}_{\text {. On Admission }}$
No. 6

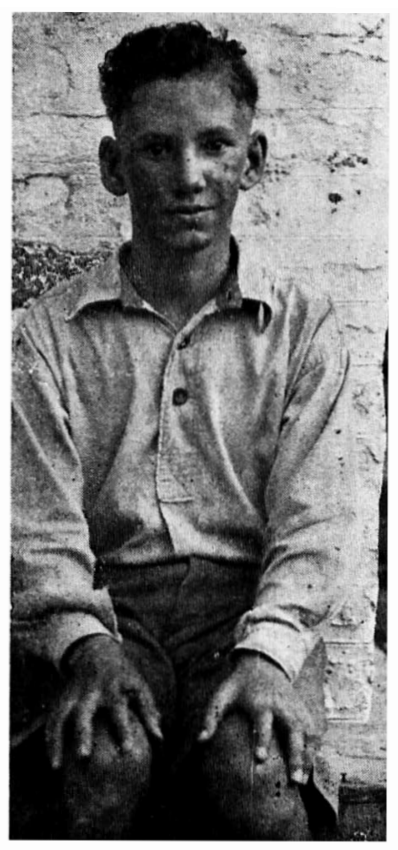

After 18 Months 
in some detail a man whom he believed to be suffering from a typical leprosy ; after briefly reviewing the occurrence of leprosy as related in history and in the scriptures, he refers to the leper hospitals that had existed in England and to large legacies that had been left for the benefit of their inmates. Thereafter, he states his views as to the causes that had almost eliminated leprosy from the British Isles in a passage that I think worth quoting. He writes, "It must therefore, in these days, be to a humane and thinking person a matter of equal wonder and satisfaction when he contemplates how nearly this pest is eradicated, and observes that a leper is now a rare sight. He will, moreover, when engaged in such a train of thought naturally enquire for the reason. This happy change, perhaps, may have originated and been continued from the much smaller quantity of salted meat and fish now eaten in these kingdoms; from the use of linen next the skin; from the plenty of better bread; and from the profusion of fruits, roots, legumes and greens, so common in every family." White then enlarges upon this subject, but the above passage contains his essential points, and considerations of time forbid further quotation.

When it has been concluded that a patient is suffering from leprosy, it becomes necessary to recognise the type of his disease, whether neural or cutaneous and, if the latter, whether he has also neural manifestations which, if present, may be primary when they are evidence of a still active pathological process, whether progressive or retrogressive, or they may be secondary when they are the result of past nerve involvement ending in nerve destruction. Next, in respect of cutaneous manifestations the stage of his cutaneous leprosy has to be determined, whether it is in the first stage, presenting lesions which are not anæsthetic and which contain few $M$. leprae, or in the second stage with the appearance of nodular lesions or of diffuse infiltrations containing numerous organisms, or in the third stage when elimination has set in and the lesions are obsolescent. Then the phase of the disease has to be determined, whether it is quiescent, of which the principal characteristic is the absence of constitutional disturbance; or, it may be reactionary, characterised by constitutional disturbance associated with exacerbation of old lesions and the appearance of new lesions; these manifestations, in the first and second stages of cutaneous leprosy, remain after the cessation of the constitutional disturbance, but in the third stage, that is the stage of elimination, they disappear as the constitutional disturbance subsides, often with marked 
improvement in the patient's condition ; or, again, the phase of resolution may be present in which a return from the reactionary to the quiescent phase is taking place.

The observations bearing on the patient's leprosy that I have just outlined have their ultimate importance. Of no less moment and of more immediate concern is a very strict search for any predisposing causes that may be present. The need for searching investigation has to be emphasised, because the patient's leprosy is too often allowed completely to overshadow other conditions from which he is suffering, though the overlooking of these is likely to have far reaching repercussions upon his prospects. Such other conditions may not be obvious. Thorough physical examination is essential, as also are routine examinations of the urine, of the faeces for ova and cysts, and also of the blood including hæmoglobin estimation; further, the performance of the Kalın Test is necessary and that of the Wassermann Test is desirable. Determination of the erythrocyte sedimentation index will be needed, but usually at rather a later stage. Further, I would stress the importance of the appraisement of the results of treatment, in due course, by laboratory methods, in addition to the previous application of these means for diagnostic purposes.

By the above procedures one or more unfavourable but perhaps rectifiable factors will usually have been brought to light. The work is rather laborious, but it is fully repaid by results, while its thorough performance forms the very foundation of success; and the rectification, in so far as possible, of unfavourable conditions is the first step in treatment.

The thorough treatment of any unfavourable intercurrent conditions is as essential as is that of persistent predisposing conditions. Leprosy per se is not a cause of severe debility unless the disease is very advanced. Patients are not infrequently seen who may fairly be described as fit persons who have the misfortune to harbour $M$. leprae in their tissues ; it is those who are in or who can be brought to such condition who may benefit from more particularly anti-leprotic measures, while any application of such measures to patients in poor condition leads to certain failure.

It is difficult to over-estimate the importance of the recognition and of the thorough treatment of persistent conditions that have given the opportunity for $M$. leprae to establish itself and flourish in the patient's tissues. Sometimes, however, a patient is found in whom the predisposing 
cause has been transient, and that patient is likely to do well. Sometimes some condition is found whose very treatment is apt to aggravate the patient's leprosy; for instance, many advanced lepers who are also suffering from syphilis, do badly on organic arsenical compounds, while potassium iodide in doses appropriate to syphilis may prove disastrous to the patient. Again, the prolonged presence of some predisposing cause may have brought the patient to a condition such that no treatment will restore him sufficiently for him to benefit by anti-leprotic measures, as happens not infrequently where ankylostomiasis of longstanding and of great severity is present. Further, some condition may be found which is in itself a contra-indication for anti-leprotic measures as, for instance, chronic nephritis or pulmonary tuberculosis.

The question of more particularly anti-leprotic measures arises when the patient is already in, or has been restored to good general condition. The results of physical examination, of the laboratory examinations to which reference has already been made, and of the determination of the Erythrocyte Sedimentation Index, show when this condition is attained.

It may be well here briefly to consider the determination of the sedimentation index and its bearing on prognosis and on treatment.

A thorough mixture of four parts of the patient's blood with one part of a five per cent. solution of sodium citrate is drawn up into a sedimentation pipette which is placed vertically in a stand. The length of the column of citrated blood is measured ; then, at the end of one and a half hours and again at the end of two and a half hours, the amount of sedimentation that has taken place is measured, and the mean of these two readings is expressed as a percentage of the length of the column of citrated blood. The sedimentation index so determined is an indication of the degree of debility from whatever causes from which the patient is suffering. In the extremely debilitated the sedimentation index is very high, and the less the patient's debility, the lower is his sedimentation index. In leprosy, with one or more concurrent conditions present, the sedimentation index is a measure of the gross amount of debility and, as the effects of the concurrent conditions are eliminated under treatment, the sedimentation index falls. Further, the sedimentation indices in uncomplicated quiescent leprosy have been ascertained with sufficient accuracy to be helpful. In normal man, or in the ex-leper with or without residual 
deformity, the sedimentation inclex is about 10 , and it is but little higher in primary neural leprosy ; in early cutaneous leprosy the sedimentation inclex is below 20 ; in more heavily involved cases it is below 30 ; and in very heavily involved cases it is below 50 .

Approximate only though these figures are, they are of very great value in determining treatment. Thus, if a patient is found to have a higher sedimentation index than is appropriate for his stage of leprosy, then the following possibilities are indicated:- (a) That concurrent conditions have not yet been successfully treated, or that, perhaps, they have been missed. (b) That the patient may be in the phase of reaction; and here the determination of the sedimentation index is of particular value, since it begins to rise before other signs become manifest.

It is important, however, to remember that when antileprotic measures are being taken that involve the production of the least clinically recognizable reactions at short regular intervals, the sedimentation index, if determined within a day or so of treatment, will be higher than the figures that I have given which apply to quiescent leprosy, but that thereafter it should rapidly fall to the quiescent figure; and, further, that as favourable progress is made, the sedimentation index in the quiescent phase will itself fall.

The sedimentation index should first be determined when the patient's general condition gives reason to suppose that he may now benefit by more particularly anti-leprotic measures; supposing his sedimentation index shows him to be in such condition, these measures are instituted, and the sedimentation index should subsequently be determined as a matter of routine at quarterly intervals, and at other times when there is lack of progress without obvious cause, or when it is wished once more to begin anti-leprotic measures after they have for any reason been discontinued.

The immediate object in treatment, as regards the patient's leprosy, is to expose to dissolution such small numbers of mycobacteria as the patient's general defensive powers can destroy, and to enable specific immunity gradually to develop. In primary neural leprosy, general treatment may be all that is necessary; but, if not, then anti-leprotic measures are instituted; extreme care is necessary, or dissemination of $M$. leprae and extension of the disease will result.

In early cutaneous leprosy it is desired to produce very slight and transient evidence of reaction at short regular intervals, manifested by a rise of temperature of a degree or 
so associated with appearances of slight activity in existing lesions. This slight constitutional disturbance should, at most, not last more than 36 hours; anything more than this is likely to cause the patient's condition to progress to the second stage. Similar manifestations are desired in the second stage, and injudicious treatment carries with it the risk of further extension of the disease.

In the third stage, that is in Muir's stage of elimination, treatment may be a little more energetic, since a considerable degree of specific immunity has been attained and elimination is taking place; but, even so, care must be taken not to push treatment too hard lest the patient become unduly debilitated.

I have assumed that the patient's leprosy is in the quiescent phase; should the reactionary phase be manifesting itself, then the position resembles that arising from overenergetic treatment; measures appropriate to the quiescent phase are now contra-indicated and those calculated to restore that phase are required.

The treatment of the leper, even when he is in good general condition, is beset with pitfalls. Anti-leprotic measures have carefully to be adjusted to each patient, while any attempt at mass-treatment inevitably leads to failure. The difficulty is to avoid alike the dangers of excess and the uselessness of inadequacy in treatment. Much is done to attain this object by physical examination of the patient, and more if his temperature is regularly recorded and if his erythrocyte sedimentation index is frequently determined; by these means unfavourable conditions are detected and can often be dealt with before they have done much harm.

There is, undoubtedly, much disappointment with the results of treatment. Many failures are due to the fact that the patients concerned have not been, and were perhaps incapable of being restored to such general condition as would enable them to benefit by anti-leprotic measures. Patients who cannot be so restored should frankly be recognised ; to submit such to prolonged anti-leprotic treatment wastes the time of those responsible for their care and does them no good ; they become very understandably dissatisfied, and their discontent spreads far and wide, causing other and perhaps more hopeful lepers to refrain from coming forward. It is far better to concentrate upon the more promising cases, since every leper whose disease under treatment has undergone arrest is a propagandist whose very presence in a population whose leper-content can as yet 
only be guessed at, will encourage the early lepers, who alone respond fairly readily to treatment, to reveal themselves.

Adequate treatment of lepers is time-consuming, and in established leprosy, results are attained but slowly. The hopeful fact remains that an increasing amount of success is being won even in fairly advanced cases, while the more attention is paid to the patient's general condition and all that implies, the greater the probability of arrest of his leprosy. 